\title{
Beyond anti-PCSK9 therapies: the potential role of resistin inhibitors
}

\section{Amirhossein Sahebkar}

In his interesting News \& Views article (Davidson, M. H. PCSK9 antibodies: a dividend of the genomics revolution. Nat. Rev. Cardiol. 10, 618-619 [2013]), ${ }^{1}$ Davidson discussed the successful experience with strategies to inhibit proprotein convertase subtilisin/kexin type 9 (PCSK9), particularly using monoclonal antibodies (mAbs), and the value of this therapeutic approach as an example of translational research from genomics. PCSK9 mAbs (such as AMG145 and REGN727) have shown promise in randomized, controlled trials, ${ }^{2-5}$ and are currently being investigated in long-term clinical outcome studies. ${ }^{6,7}$ Interestingly, percentage reductions in levels of LDL cholesterol with PCSK9 mAbs reported in phase I and phase II trials are the largest ever achieved by a pharmacotherapeutic agent. ${ }^{8,9}$

I would like to highlight a finding that could potentially be applied in the design of novel LDL-lowering agents with efficacies beyond even that of PCSK9 mAbs. Resistin, a small, cysteine-rich protein secreted from macrophages and adipose tissue, has been shown to stimulate PCSK9 by enhancing gene expression and stability of the protein, leading to the downregulation of the LDL receptor. ${ }^{10}$ Enhancement of PCSK9 expression by resistin is mediated via sterol regulatory element-binding protein 2 (SREBP2) induction, and is accompanied by increased 3-hydroxy-3-methylglutarylcoenzyme A (HMG-CoA) activity. Interestingly, the stimulating effect of resistin on LDL-receptor expression is not exerted exclusively via PCSK9, given that a significant reduction in LDL-receptor expression has been observed after resistin treatment in PCSK9-knockout mice. ${ }^{10}$ This effect strengthens the hypothesis that resistin might directly interact with, and promote lysosomal degradation of, the LDL receptor, because resistin and PCSK9 have a notable structural similarity in the cysteinerich C-terminal domain. ${ }^{11}$ This point also raises the possibility that PCSK9 mAbs might interact with resistin, but whether such an interaction exists and accounts for part of the LDL-reducing capacity of mAbs has not yet been tested. Finally, resistin treatment has been proposed to blunt the response to statin therapy by dramatically reversing statin-mediated upregulation of hepatocyte LDL receptors. ${ }^{10}$

These biological mechanisms open avenues for research into resistin inhibition as a potentially effective LDL-lowering strategy in severe types of dyslipidaemia, such as familial hypercholesterolaemia, as well as in patients who are statin intolerant or nonresponsive to these drugs. The efficacy of resistin inhibitors might be expected to be even higher than that of PCSK9 mAbs. As discussed, PCSK9-independent mechanisms are involved in the resistin-mediated regulation of LDL metabolism. Moreover, inhibition of resistin might be accompanied by a plethora of pleiotropic effects owing to the involvement of this adipokine in inflammation, thrombosis, angiogenesis, and endothelial dysfunction. ${ }^{12}$ Inhibition of resistin might also prevent hepatic steatosis and nonalcoholic fatty liver disease by inhibiting HMG-CoA and lipogenesis, and could serve either as an adjunct (to enhance the efficacy) or as an alternative (in patients with refractory dyslipidaemia or statin intolerance) to statins. Future studies are needed to verify this untested hypothesis by evaluating the LDL-lowering potential and other cardiovascular benefits of resistin inhibitors.

Biotechnology Research Center, Mashhad University of Medical Sciences, Mashhad 9177948564, Iran. sahebkara@mums.ac.ir

\section{Competing interests}

The author declares no competing interests.
1. Davidson, M. H. PCSK9 antibodies: a dividend of the genomics revolution. Nat. Rev. Cardiol. 10, 618-619 (2013).

2. Sullivan, D. et al. Effect of a monoclonal antibody to PCSK9 on low-density lipoprotein cholesterol levels in statin-intolerant patients: the GAUSS randomized trial. JAMA 308, 2497-2506 (2012).

3. Raal, F. et al. Low-density lipoprotein cholesterollowering effects of AMG 145, a monoclonal antibody to proprotein convertase subtilisin/ kexin type 9 serine protease in patients with heterozygous familial hypercholesterolemia: the reduction of LDL-C with PCSK9 inhibition in heterozygous familial hypercholesterolemia disorder (RUTHERFORD) randomized trial. Circulation 126, 2408-2417 (2012).

4. McKenney, J. et al. Safety and efficacy of a monoclonal antibody to proprotein convertase subtilisin/kexin type 9 serine protease, SAR236553/REGN727, in patients with primary hypercholesterolemia receiving ongoing stable atorvastatin therapy. J. Am. Coll. Cardiol. 59, 2344-2353 (2012).

5. Roth, E. M. et al. Atorvastatin with or without an antibody to PCSK9 in primary hypercholesterolemia. N. Engl. J. Med. 367, 1891-1900 (2012).

6. US National Library of Medicine. ClinicalTrials.gov [online], http://clinicaltrials.gov/show/ NCT01663402 (2013).

7. US National Library of Medicine. ClinicalTrials.gov [online], http://clinicaltrials.gov/show/ NCT01764633 (2013).

8. Dias, C. et al. Effects of AMG 145, a fully human monoclonal antibody against PCSK9, on low-density lipoprotein cholesterol in subjects taking statins: a phase I, randomized, doubleblind, placebo-controlled, ascending multipledose study [abstract 923-4]. J. Am. Coll. Cardiol. 59, E1379 (2012).

9. Stein, E. A. et al. Effect of a monoclonal antibody to PCSK9 on LDL cholesterol. N. Engl. J. Med. 366, 1108-1118 (2012).

10. Melone, M., Wilsie, L., Palyha, O., Strack, A. $\&$ Rashid, S. Discovery of a new role of human resistin in hepatocyte low-density lipoprotein receptor suppression mediated in part by proprotein convertase subtilisin/kexin type 9. J. Am. Coll. Cardiol. 59, 1697-1705 (2012).

11. Hampton, E. N. et al. The self-inhibited structure of full-length PCSK9 at $1.9 \mathrm{~A}$ reveals structural homology with resistin within the C-terminal domain. Proc. Natl Acad. Sci. USA 104, 14604-14609 (2007).

12. Jamaluddin, M. S. et al. Resistin: functional roles and therapeutic considerations for cardiovascular disease. Br. J. Pharmacol. 165, 622-632 (2012). 